\title{
Assessing the Risk of the Evolution of Resistance to Pesticides Using Spatially Complex Simulation Models
}

\author{
M.A. CAPRIO ${ }^{1}$, N.P. StORER ${ }^{2}$, M.S. Sisterson ${ }^{3}$, S.L. \\ PECK ${ }^{4}$ AND A.H.N. MAIA
}

'Department of Entomology and Plant Pathology, Mississippi State University, Mississippi State, Mississippi, USA, ${ }^{2}$ Dow AgroSciences LLC, Indianapolis, Indiana, USA; ${ }^{3}$ San Joaquin Valley Agricultural Sciences Center, Parlier, California, USA; ${ }^{4}$ Department of Biology, Brigham Young University, Provo, Utah, USA; ${ }^{5}$ Embrapa Meio Ambiente, Jaguariuna, SP, Brazil

\subsection{Introduction}

As a class, insects have an extraordinary ability to adapt to diverse environments (Chapman, 1982). At the species level, the life system characteristics typically shared among agricultural pests - the ability to rapidly colonize new or unstable habitats through rapid reproduction and a high motility - include an ability to respond to changing environments (Kennedy and Storer, 2000). Insecticides, presented through either transgenic crops or conventional means, represent a severe environmental challenge in otherwise suitable habitats and pose an interesting evolutionary problem to pest species. Individuals with a genetically based ability to overcome these challenges have higher fitness in more environments than other individuals in the population, and their genotypes should increase in frequency, leading to the evolution of resistance to the toxin. After the first documentation of insect resistance to a synthetic insecticide in 1947 (Metcalf, 1973), the process of resistance evolution quickly drew the interest of population geneticists (Crow, 1957). A desire to proactively manage resistance propelled development of an understanding of the underlying population genetics of resistance evolution. The basic principles of modern resistance management programmes were developed in a series of papers by Taylor and Georghiou (Georghiou and Taylor, 1977a,b; see also Taylor and Georghiou, 1979, 1982, 1983; Taylor, 1986). These papers clearly identified the driving roles of functional dominance of resistance, including the effects of pesticide dose and the problems posed by pesticide decay, and the rate of immigration of susceptible 
insects in determining rates of adaptation. These concepts were further developed by Tabashnik and Croft $(1982,1985)$ and others (Curtis et al., 1978; Plapp et al., 1979), who demonstrated that consideration of multiple habitats (treated and untreated fields) is important in understanding the evolution of resistance. They used a continent-island concept in which there is an influx of susceptible alleles but negligible migration of resistance alleles into the untreated population. This concept essentially envisages an infinite supply of susceptible immigrants, whereby the movement of any resistant individuals out of the area of selection would have an insignificant impact on gene frequencies in the surrounding habitats. Comins (1977a,b, 1979) developed a two-patch model that relaxed the assumption of outward migration and allowed the diffusion of resistance alleles into untreated habitats. He concluded that density-dependent processes in population dynamics in each patch are critical components of any multi-patch model and therefore that infinite population models have limited ability in predicting resistance evolution. This idea was further explored by Gould et al. (1991), Onstad et al. (2001), Storer et al. (2003b), Sisterson et al. (2004) and Crowder and Onstad (2005), for example.

The development of spatially explicit finite population models (Caprio and Tabashnik, 1992; Mallet and Porter, 1992; Caprio, 1994; Peck et al., 1999; Sisterson et al., 2004) suggested that moderate rates of gene flow, interacting with genetic drift, could actually cause faster resistance evolution than either high or low rates of gene flow under some conditions. Spatial effects and heterogeneous distributions of treated and untreated patches could also impact resistance evolution rates (Peck et al., 1999; Storer, 2003; Sisterson et al., 2005). For the first time, these spatially explicit stochastic models produced estimates of variation due to demographic stochasticity

From simulation studies using the early models, the conclusion that pesticide dose is a key determinant of rates of resistance evolution highlighted a restriction to devising effective resistance management strategies. While there is the potential for immigration to delay resistance evolution when high doses of an insecticide are used, this strategy was unlikely to be successful with conventional insecticides, because the uneven application and continuous decay of those compounds would expose insects to more moderate doses (Taylor and Georghiou, 1982), altering the dominance of resistance. Host plant resistance factors that are expressed at high leveis throughout a crop plant are able to overcome this restriction and open up new avenues for resistance management (Gould, 1986). The advent of transgenic crops that constitutively produced insecticidal proteins accelerated the need and ability to devise effective management strategies (McGaughey and Whalon, 1992: Roush, 1994; Wearing and Hokkanen, 1995; Gould, 1998). Gould (1994) and Roush (1994) argued that combining a consistent high dose in the genetically modified plant with planting of plants that do not express the toxin (a refuge from selection) could delay the evolution of resistance dramatically. The concept that the plants would continue to express the toxin in sufficient quantities over the course of the season to avoid large changes in functional dominance was key to this proposal (Onstad and Gould, 1998). Initially. mixtures of transgenic and non-transgenic seeds seemed like an ideal method to implement this strategy (Tabashnik. 1994a). but 


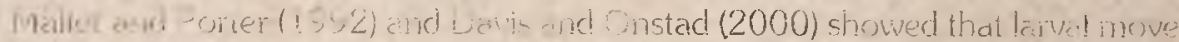

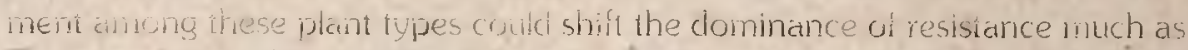

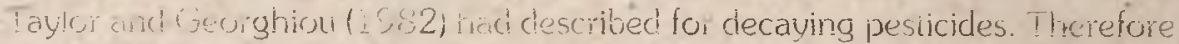
the planing of struciuted refuges mas required when insecticidal transgenic ciops

the evolution of resistance is c population genetics phenomenon. affecied bij crmilo interations amona pret hiology and ecology propertie

ticide and pesticide use patterns (Georghiou and Taylor. 1977a.b): and models have alivays played an important ole in our understanding (Taulor. 1983. Tabashnik 1986). However in a broader sense. all humans construct models Indeed t has been suggested that human knowledge can be seen as the con struction of models to understaind our social. physical and biological surroundings (Richmond. 2001). Conside the simple everyday example of the decision to make a up of coffee or tea in the morning. One might evaluate the time and difficultu involved. the cost of the beverage. how much better it tastes than the coffee or tea at work. how much work is waiting at work. etc. Normally. one would not fomalize all these paimeiers, but they have probably been consicieied. at 'easín leetingly, by many ai one time or another. Similarly. those who work in agricultural systems have conceptual models of what happens in those systems and what inpuits oi parameters are most important, and. in most cases. have not formalized values for those parameters. While there are many different goals of modelling. one goal is to formalize these conceptual models. to explicitly state the rules and relationships between the components of the models. The model allows one to organize all available data into a coherent framework with clearly stated rules regarding transformation of the model from one state to the next. Once constructed, these models allow one to test hypotheses about effects of changes in parameters or how different components interact and result in observed system behaviour. Models based upon mechanistic processes become experimental systems in which it is possible to develop hypotheses about how one expects the system will behave and then test the system response (Peck. 2004). In many cases, the system is complex enough that it can behave in unexpected ways, and the conclusions are not necessarily an obvious result of the assumptions and rules used in model construction. Indeed, it is often when the model behaves differently from expectations that it is providing the most important information. Such unexpécted results may indicate that something is missing from the model, they may guide future research by identifying research needs, or they may indicate that our conceptual understanding of the system is incorrect and requires modification. For example, one early assumption of the high-dose/refuge strategy was that random mating between adults produced in refuges and transgenic fields would maximize delay of resistance evolution (Tabashnik, 1994b). Using a spatially complex model, Caprio (2001) suggested that because of source-sink dynamics, some degree of isolation between a refuge and a highly toxic crop might actually delay resistance much longer than having random mating between the different habitats. Indeed, these predictions were supported by the model of Sisterson et al. (2005), and subsequent field work demonstrated that model predictions were consistent with the patterns observed in the field (Carrière et al., 2002; Caprio et al., 2004). As noted by 
Alstad and Andow (1995), these dynamics will also create a halo of increased damage surrounding areas planted to refuges. In spatially complex models, Peck et al. (1999) and Sisterson et al. (2005). found that resistance first evolved in areas with a locally high density of transgenic fields and then spread outwards. Selection in these small areas led to foci of locally higher resistance allele frequencies, an increased frequency of resistant homozygotes and a more rapid rate of resistance evolution. Once resistance became common in these areas, migration spread resistance alleles across the region. The spatial characteristics of the system and the dispersal characteristics of the insect are critical to determining the potential for these spatial dynamics effects (Sisterson et al., 2005).

\subsection{Simple Mersus Complex Models}

As we have seen, there is a wide range in complexity of resistance models, from elegantly simple analytical models to increasingly complex simulations of specific agroecological systems. The simplest models include only a few parameters: (i) initial resistance allele frequency: (ii) strength of selection (e.g. percentage of the population exposed to the insecticidal transgenic crop); and (iii) the dominance of resistance. Because such models have only a few parameters, it is possible to conduct a complete analysis, which provides an understanding of the role of each parameter. It is often possible to construct analytical solutions to identify optimal strategies (e.g. Lenormand and Raymond, 1998). From these simple models, we have developed an understanding of the general principles of resistance evolution from which we can design effective resistance management strategies, such as the potential power of the high-dose/refuge strategy (Gould. 1998). For example, the non-random mating model developed by Caprio (1998), a simple deterministic model with a few parameters, was used for designing preliminary proactive resistance management strategies for Bt cotton in the mid-west region of Brazil (Fitt et al.. 2006).

However, these models do not inform us about the effectiveness of a specific strategy in a specific system. While simple models provicle an understanding of the few parameters that are useci. it is unclear how dependent the results are on many of the simplifying assumptions These models ara highly abstracted and simplified versions of field conditions, and the input parameters may actually be combinations of many other parameters that can be measured empirically. Because of this simplification/abstraction process, there may be many underlying processes not explicitly represented. Therefore, it is not possible to evaluate properly the influence of those hidden parameters on the resistance model end points. Of course, even complex models are also simplified/abstracted versions of reality and suffer the same problem. presumably to a lesser extent.

At the opposite end of the spectium are complex: models that may represen. several interrelated complex process and incoiporaic t: is. if not hundrecis, of parameters. These paraneters tend io be less abstraci and closer to parameters for which compirically measured estimates exist. althrugh data gaps in each model certainly exist. They end ir he morm highly me hanistic simulations of

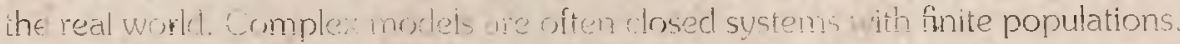




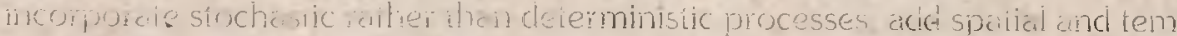

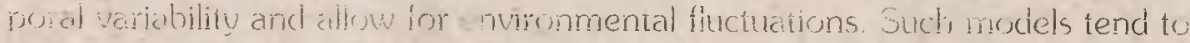
be much more susien-specific coaling with a particular pesi. crop or gerography convilation ie.g. Peck ei al. . 95\%: Guse ei al. 200z. Storer ei al 2003a: Gisierson ei al. 2u(1) 4 . While ihese modek have noi radically altered our un veistanding of resistance evonulion - the same parameters and the came resistance inanagement shiegies have been supported as were supported bu simpler. analutical models - the permii insights into the actual risk of resistance in any giron :eal-world scencinio as well as into the potenicil effectiveness and imitaituns of a given management laciti:

Is an example landscaps ecology is increasingly being integrated into restrince siudies. Spatially rich models allow one io incorporate insect movement among inultiple habiais to e:amine issues such as interactions among differmi cops (Giorer ei al. 2.,03a,b). Tre key component in such models is a shif in iocus from a singie msect on a single crop to a broader perspective of following an inseci as it moves through a variety of habitats during the course of a giowing season. "hese models can include temporal, as well as spatial, variation in such components as crop composition and toxin expression. For example, Helicoverpe zeo in the Delia region of the mid-southern USA begins the season on wild hosts such as crimson clover and wild geranium (Etadelbacher, 1979: Parker. 2000). Later, it moves on to whorl and eventually eai-siage maize. By mid-July. most maize is no longer suitable habitat for $h$. $z e c$, and, at this time, adults may move into a number of crops, including cotton. sorghum and soybean. Finally, in late summer, some H. zea may overwinter in crop fields (where cultivation may yield a significant amount of mortality), while others may move to weedy hosts and overwinter there (Parker 2000: Storer et al. . 2003a). Figure 4 . I shows simulated numbers of pupae in each habitat over the course of a season (Parker and Caprio, unpublished data). Population dy namics in each of these habitats. combined with population genetic issues, such as selection by various pesticides and toxins in the different habitats, will affect the impact of pest management decisions. For example, while high adoption rates of transgenic cotton in the Delta may reduce the number of $H$. zea moths emerging from the cotton acreage and reduce overall density in the autumn, density-dependent interactions in maize may allow $H$. zea numbers to build back up to damaging numbers in the early season when maize is a host. Numbers are limited in ear-stage maize, as generally only one adult will emerge from each ear due to cannibalism (Barber, 1936). Thus, density-dependent mortality will be high at this stage when populations are large (which can accelerate resistance evolution) (Storer et al., 2003a), but if population levels decline because of mortality due to transgenic crops, density-dependent mortality may have less impact on the evolution of resistance. When density levels are high, each ear of maize will probably be infested with multiple larvae. H. zea larvae are cannibalistic, and the largest larvae are most likely to survive. When a $B t$ toxin is present in kernels, resistant larvae will experience less growth retardation than susceptible larvae and will have a greater chance of surviving not only the $B t$ toxin, but also the density-dependent mortality. In contrast, when densities are low, individual ears will likely be uninfested or have a single larva. and the rate of selection will 


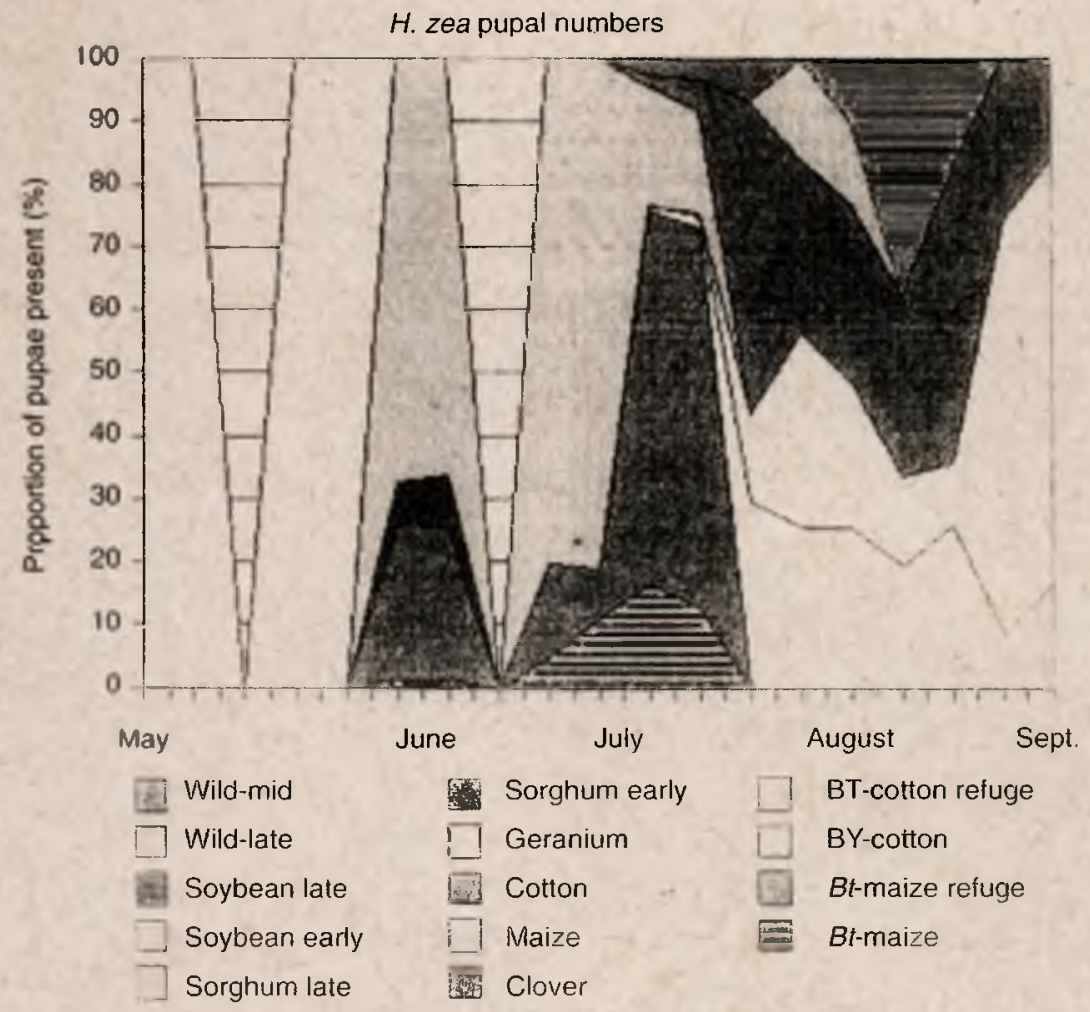

Fig. 4. Termporal dynamics of Helicoverpa zea pupal numbers in a simulated midsouthern US agroecosysiem. The $y$-axis is the proportion of all pupae present on a given date. (Caprio and Parker, unpublished data.)

cnly be related to the chance of surviving the Bt toxin. These dynamics demonstrate that the wwo systems interact in a complex manner, and both systems must be undersiood before one can begin to assess the risk of the evolution of resistance. Current transgenic maize varieties express Cry $1 \mathrm{Ab}$ or Cry IF insecticida! proteins from Bacillus thu!ringiensis $\langle B t\rangle$ to conirol Lepidoptera: while Cry $1 \mathrm{AC}$ and combinations of Cry Ac with Cry If or Cry2Ab are expressed in current transgenic cotton varieties. Several studies have demonstrated the potential for some level of cross-resistance among these toxins (e.g. Gould et al. 1995a: Jurat-Fuentes et al. 2000; Siqueira et al. 2004). The overall rate of the evrlution of resistance to the Cry proteins in cotton will be impacted by pest management decisions in maize (II Si/HESI. 1999: Storer et al. 2003a).

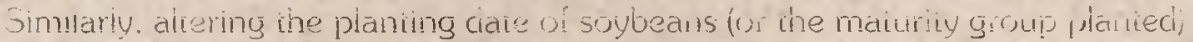
can affect the infestation of these habitats which act as refuges to tran: genic cuitui! (Gustafon ei al 21,00). Clearly the interactions ainong the temporal dynamics of the tifferent habiats in a system may also play an impor

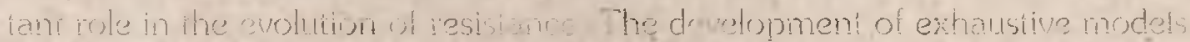

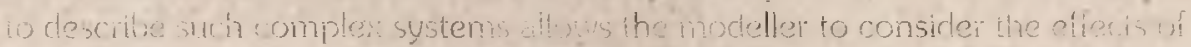

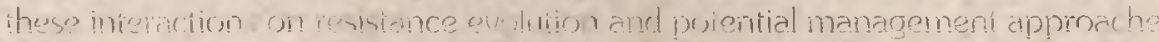




\subsection{Resistance Probabilistic Risk Assessment}

The possibility of resistance evolution should be regarded as a hazard associated with the use of any pesticidal technology, similar to the possibility of water, soil or food contamination. The challenge when deploying these technologies responsibly is not just to determine that such hazards exist, but to quantify the risk; that is. quantify the probability of an adverse occurrence and the magnitude of the effect of that occurrence. Where an unacceptable risk is identified, risk mitigation measures (such as spray drift reduction or pre-harvest intervals) can be implemented. Leonard (2000) describes the application of the risk assessment paradigm to resistance management for pesticides in Europe and a set of guidelines for performing the risk assessment. Beyond such qualitative approaches, complex models can be used to determine the magnitude of the risk quantitatively and to design appropriate mitigation measures.

\section{Modelling uncertainty}

Uncertainty is inherent in all risk assessments in which simulation models are used to extrapolate information beyond the domain of direct observation (Hoffman and Kaplan, 1999). Different types of simulation models will produce a variety of different results, and interpretation of these results will depend on the underlying structure of the models and the assumptions (explicit and implicit) made. For complex models, interpretation becomes far more challenging as multiple processes are occurring simultaneously, and there is a temptation to use the models in a predictive manner. In quantitatively assessing the risk of the evolution of resistance, information on mean values of selected end points (such as time until resistance allele frequency exceeds some level over some proportion of the Bt crop area) is not sufficient. In some cases (skewed or multimodal output distributions) even additional summary measures, like variance or standard error, do not allow risk quantification. To be useful, the model output information should be represented by Monte Carlo probability distributions, used for calculating the probability of occurrence of adverse effects, generally related to the tails of output distributtions (see Fig. 4.2). For a given set of input parameters, deterministic models will always produce the same point estimate, therefore not being useful for estimating risks. From a broader perspective, when assessing risk we are most often interested in the tails of probability distributions, in particular the tail that represents the proportion of events that exceed our acceptable criteria. The mean of the distribution, which has been the focus of resistance modelling since its inception, may be relatively uninformative, especially when the shapes of the Monte Carlo distributions of alternative risk scenarios are different. It is conceivable that two strategies with similar mean times to resistance could actually have different risk assessments, because they respond differently to conditions that are responsible for 


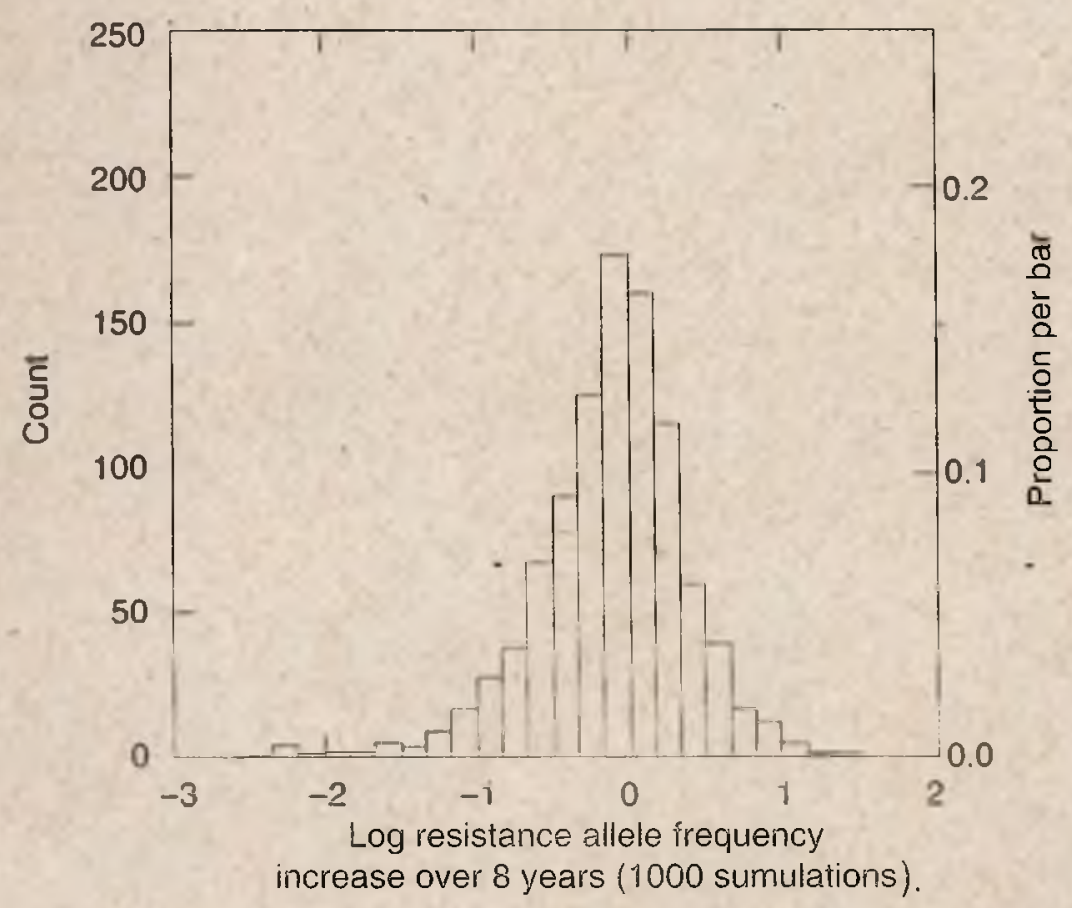

Fig. 4.2. ivionte Carlo probability distribution of the mean relative log raie of increase for a resistance allele to a high-dose iransgenic eveni with fitness cosis over the initial 8 years of the simulations.

the tails of the distribution, such as rare draws of relatively high values of dominance.

Generally. if one wants to know how sensitivo these results are to isolate or conjoint perturbations in the initial input parameters, one runs the model for a series of parameter values without assigniing probabilities io those values the socalled sensitivity analysis. The aim of sensitivity analysis is not producing risk estimates, bit evaluaing the relative influence of input parameiers on the model end points. It is useful for indicating that information on some key parameters should be refined, while, for other ones, improving information would be irrelevant (Isukapalli and Georgopoulus. 2001). An approach widely used for re sistance risk assessment is based on choosing a series of likely or unlikely scenarios (best-case, worst-case. etc.) by sefting input parameters to scme user-

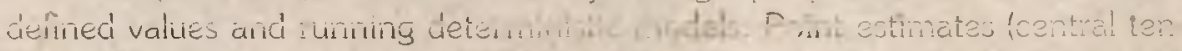
dency measures, like mean) are then produced for each output variable without

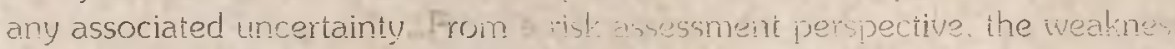
of this approach (deterministic scenario, arialysis) is that ao probabilities are assigned to the different scenairs. Gr conarios may be seen as unlikol:

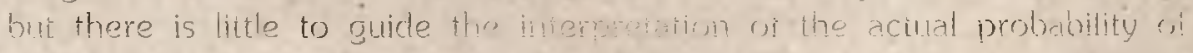




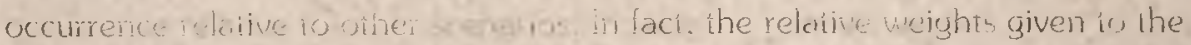
different scenarios are entirelu whim ine and vary considerably anong users of the information. Despite that. it niturnes of model whs with such settings are meaningless for understanding the al world unless there is some way to assess the probability of such a worst case occurring. These results can be easily misinterpreted as being approprictucluc conservative whein ciesigning a resistance managenent plan or setting prilicy

To be useful foi risk assessments models need to produce probabilistic outputs represented by distributions for each end point of interest. These distri butions are used to quantify iisks generally related to the occurrence of extreme events. Because they are increasingly based on mechanistic piocesses reflecting real world biology. complex models lend themselves to be used as predictive tools. just as climate models are used to predict future states of climate. and epidemiological models are used to predict the spread of new diseases. However. none of these models should be used to provide exact predictions, since there is considerable uncertainty inherent to their underlying process. To be used as predictive tools it is imperative that such models be analysed for their inherent uncertainty. in the same way that weather forecasts incorporate uncertainty by stating there is a certain chance of rain and providing a range of likely amounts. represented by the rain output distribution. For complex models that incorporate stochastic variability and parameter uncertainty, both sources of variability are important in performing risk assessments for the evolution of resistance. We see the formalization of methods for probabilistic risk assessments for transgenic crops using uncertainty analysis as an important new direction in the modelling of resistance evolution. If we are to progress beyond the conventional risk assessment of transgenic crops in which a series of scenarios (best-case, worstcase, etc.) are simulated using deterministic models with no formal guidance on the likelihood of those-scenarios, then we must seek to formalize methods for accounting for parameter uncertainty, as well as process stochasticity. These methods allow production of model outputs represented by probability distributions, instead of simple point estimates. Those distributions can then be used for assessing risk, which, by its own definition, should incorporate the probabilistic nature of adverse event predictions. Below we present parameter re-sampling procedures based on Monte Carlo methods that, when combined with stochastic resistance models, will provide an alternative to deterministic scenario analysis.

Uncertainty in model predictions arises from several sources. As insect resistance simulation models seek to simplify highly complex ecological systems in highly variable environments, it cannot be known that the model structure sufficiently, or accurately, describes the system being modelled. The variability inherent to the systems being modelled translates to uncertainty in the appropriate values for input variables and parameters (Fig. 4.3). The values for the input parameters are not perfectly known, and random (or unpredictable) variability in the biotic and abiotic environment (temporal and spatial) causes variability in the value of the parameters. Finally, for a stochastic model of a closed system, different outcomes can result from the same inputs (Fig. 4.4a). The modeller is therefore challenged to account for and describe the uncertainty and variability. 


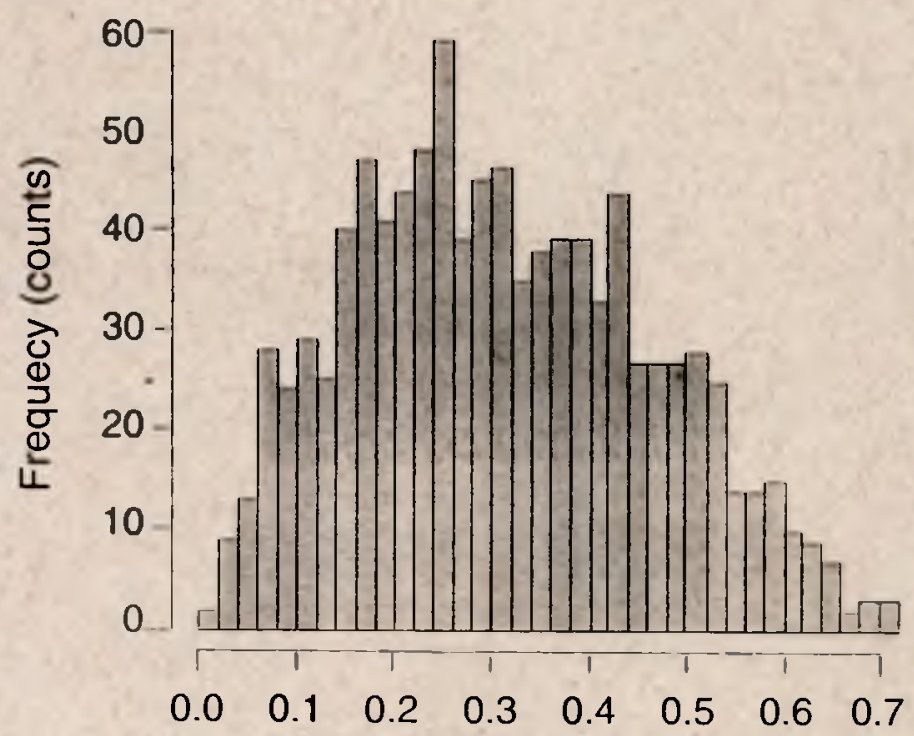

Fitness costs

Fig. 4.3. Example of a Monte Carlo probability distribution for the input parameter, Vip resistance allele fitness costs. One thousand draws were made from a beta distribution. The minimum value was 0.0 , the maximum value was 0.8 , and the most likely value was 0.2. (Caprio and McCaffery, unpublished data.)

Incomplete understanding of the biological processes leads to uncertainty in the true value of a given model parameter. Often modellers need data that have not been considered important from a pest management perspective, and therefore are unavailable. For example. differential mortality of bollworms in refuge patches compared with $B t$ crop patches during winter can have a profound effect on predicted rates of adaptation to $B t$ cotton. However, measuring the level of mortality under natural conditions is extremely difficult to do, and in the past was not regarded as important when the predominant pest management tools were curative rather than prophylactic. To measure winter mortality of bollworms. the field researcher needs to provide good estimates of the final population of pupae in the soil in the autumn and of the population of emerg ing moths in the spring. Few studies in the literature have provided these esti. mates, leaving the modeller uncertain as to the correct value to assign to this parameter.

Natural variability in the environment means that the true value of a given parameter varies with space and time To use the same example, mortality of bollworm pupae in a refuge during winter can depend on the vigour of the 


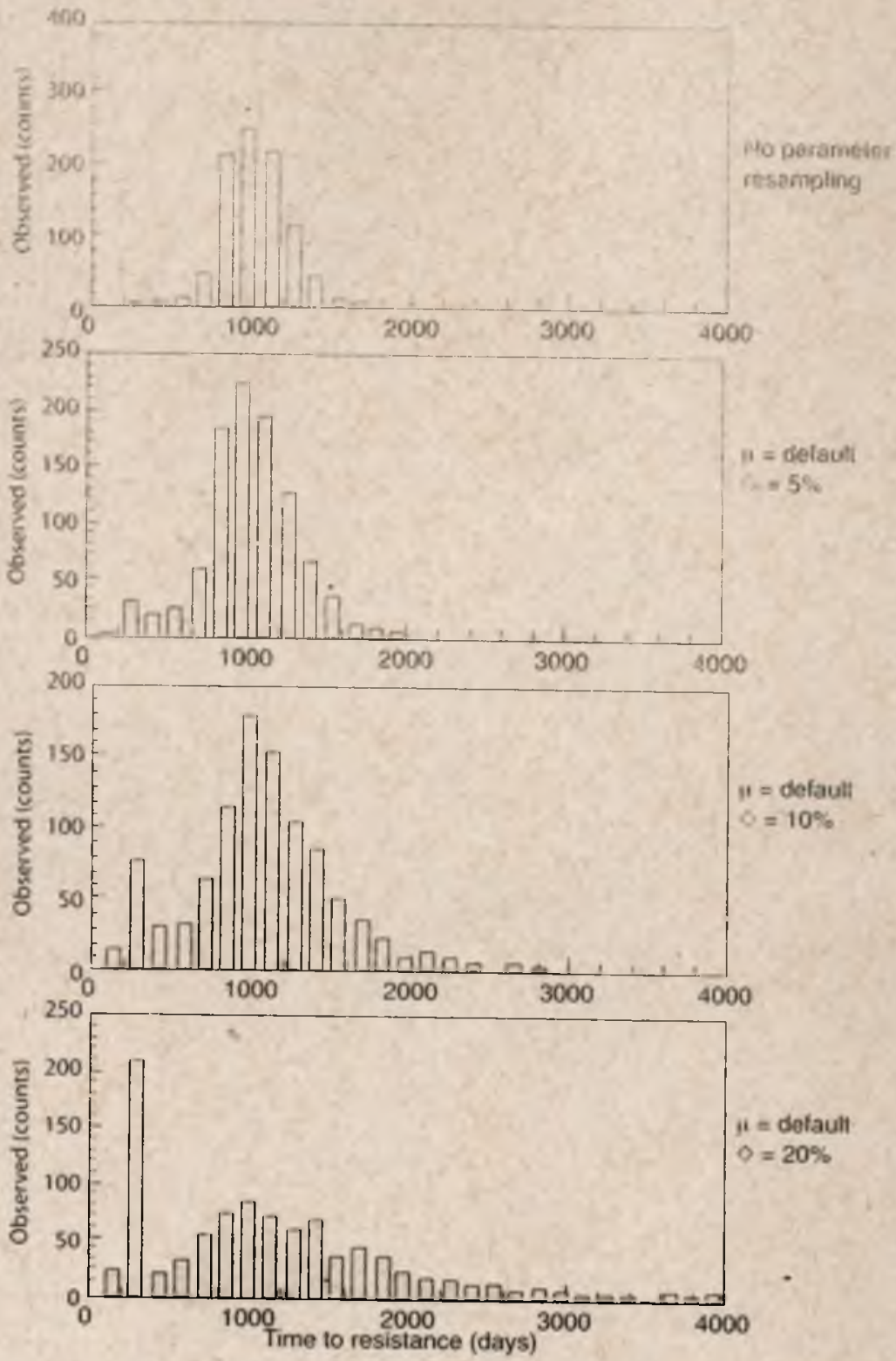

Fig. 4.4. Monte Carlo distribution for the output variable, time to resistance: (a) no parameter re-sampling, uncertainty due to stochastic variability only: (b) mean $=$ default, standard error $=5 \%$ of mean; (c) mean $=$ default, standard error $=$ $10 \%$ of mean; and (d) mean $=$ default, standard error $=20 \%$ of mean .

population as it enters winter (and therefore the quality of the host plant during the preceding larval stage), the weather conditions throughout winter (temperature, moisture), the presence of predators in the soil, the degree of parasitism of the population, the soil type and cultivation activities in the field before moth 
emergence (perhaps leading to soil compaction, or exposure of pupae to the elements). While the proportion of pupae that survive will clearly be variable, both spatially within and among fields and temporally from year to year, this complex of factors is typically modelled as a single parameter, such as winter survival (Peck et al., 1999; Onstad et al., 2001; Storer 2003; Storer et al., 2003a,b; Sisterson et al., 2004).

\section{Analysing model stochasticity}

The use of stochastic models presents an important component of modelling uncertainty, since a single set of input variables and parameter values can lead to a range in output values. The variance in model results can also be profoundly affected by stochastic influences. Deterministic models treat rates as fixed items: if the mortality rate is $60 \%$, then exactly 60 out of every 100 individuals will die during that time period, and, by extension, 60.6 die if there are 101 individuals. Therefore, a single set of input parameters gives a single set of values for output variables. In stochastic models, fractions of individuals are not allowed. and such rates are seen as the probability that an individual will die during the time period. The total number that die during any particular period would be a random draw from a binomial distribution $(N, p)$, where $N$ is the total number of individuals and $p$ is the probability. For the example above, while on average the stochastic model will give $60 \%$ mortality winter survival, any single realization could give between 0 and $100 \%$ mortality with a binomial frequency distribution of survival rates. There are a number of possible events in a model of the evolution of resistance in which such stochastic influences might be incorporated. including mortality (both natural and response to toxins), fecundity, mating and dispersal. These stochastic processes can have a large impact on model variances in some cases and negligible impacts in others (Fig. 4.4a).

In a model simulating bollworm adaptation to Bt maize and Bi cotion. Storer et al. (2003a) conducted five runs of each scenario with a particulair parameter set to improve the estimate of the mean values for the end points. Although the standard deviation stabilized after five runs, there was no way to determine whether those five runs were sufficient to capture all of the important possible stochastic variation. For example stochastic influences can be great when resistance alleles are initially rare, while they are much less important when re sistance alleles are relatively common. Selection acts independently of popula. ition size while genetic drift becomes more important as population size decreases. Extinction of resistance alleles becomes a real possibility when they are rare, so it is possible that in 99 realizations the resistant allele goes extinct

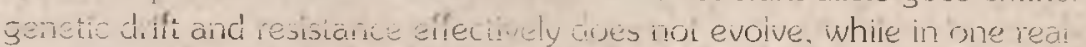
ization the resistance allele persists (and resistance may evolve very fast in this case). Cleaily the variance in these resulis is great. and questions addiessing the sate of the model become improtant if the model system was 100 -fold larger

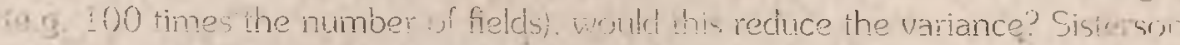

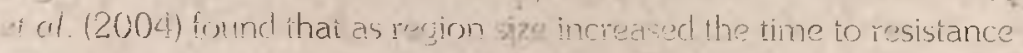




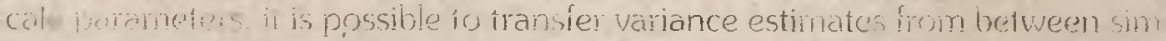

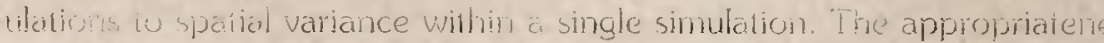

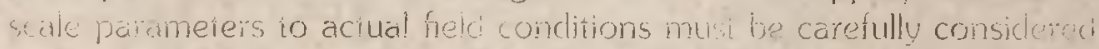
perionning risk assessments

a strochastic model is iun a sulficien number of times. The varabilitu of th: Itipul can be described by a probability clistribution. Just as it is possible lo lis: repeated realizations of deterministic models with different parameler values or ascess the impaci of uncertainty in those values on the variance in model resilit so one can use ihis technique wih a single sei of paraneters to assess the mpaci of stochasticity on the model results. One thousand realizations of a model with unvarying parameiers are likely to provide a reasonable estimate of the influence of stochastic factors on the evolution of resistance (Fig. 4.4a). While Peck et al. (1999) found that the initial distribution of $B t$ and non-Bt fields determined whether of not resistance foci became established (and therefore resisiance evolved rapidly). Storer (2003) showed in a stochastic spatially explicit model of corn rootworm adaptation to $B t$ maize that. even if the initial conditions are held constant. there can be a twofold variation in time to resistance due only io stochasticity in the model.

In this case. we assume that we know all the parameters with ino uncertainty (they remain constant for all runs), and the variance in the results reflects the limits of our ability to predict the evolution of resistance. It is therefore important to realize that, short of the impacts of scale factors we noted earlier. this distribution is the limit of our ability to predict the evolution of resistance. No additional knowledge could reduce this variance, just as no additional replication can reduce the standard deviations in a measurement

\section{Avelysing paramerer uncertainty}

Resistance modellers have traditionally attempted to understand the importance of such uncertainty by running a sensitivity analysis: by perturbing parameter values one at a time and investigating the effect of the perturbation on model outcomes. Typically, one can ascertain the effect of perturbations on the value of a parameter by applying $\pm 10 \%$ of the default value, while holding all the other parameters at their default values. By repeating this for each parameter, the- modeller can determine how important the uricertainty around each parameter is to the interpretation of model outcome. For example, Crowder and Onstad (2005) found that density-dependent mortality and functional dominance of resistance had large effects on rates of adaptation, but fitness costs did not.

The single parameter perturbation technique only allows the modeller to determine the effects of changing one parameter at a time and only under default values for the other parameters. Therefore, the technique does not allow the modeller to investigate potential interactions among pairs (or even groups) of parameters. The technique also does not allow the modeller to use information on the magnitude of the uncertainty in parameter values. There may be more information available about the true value of some parameters than for 
others, or certain parameters may be less influenced by environmental effects than others. Storer et al. (2003b) attempted to account for differential knowledge among parameters in a sensitivity analysis of their corn earworm/bollworm model by testing ranges of parameter values that represented the authors' opinion of the 'biologically reasonable' range values. The same sensitivity analysis also included investigations into potential interactions among parameters and input variables.

An alternative to the deterministic scenario approach and individual parameter perturbations that has generally been used for risk assessments is to more formally specify our uncertainty in some key model parameters. For each selected parameter, we specify a probability distribution, referred to as the input parameter distribution. In place of running a specific number of scenarios, by subjectively setting the input parameters to some values, in this risk assessment approach one runs a large number of model realizations, randomly drawing new values for each parameter from the specified input probability distributions. After completing many (often greater than 1000) realizations (replicate simulations but with randomly drawn parameters), the results can be arranged as output probability distributions for each model end point, referred to as a Monte Carlo output distribution. They are a direct measure of the impact of parameter uncertainty on the output distributions used for our assessment of risk (Fig. 4.4b. $c$, and d). One can now begin to address questions such as what is the risk of resistance evolving within a certain amount of time, or what is the probability that a transgenic event will last more than a given number of years. This description of the uncertainty of our results (which differs from our uncertainty in our parameters) is critical to effective risk assessment.

This risk assessment technique effectively assigns probabilities to outcomes and has recently been adopted to examine risk of the evolution of insect resistance to transgenic crops (Maia, 2003: Maia and Dourado-Neto. 2004; Caprio et al., 2006). Uncertainty due to environmental variability can be incorporated into the model by using a different probability each time a parameter is used cluring a single model run; for example. for each simulated winter and, in a multi-patch model, a different probability for each patch. Uncertainty due to es timates based on field data to provide the true value of a parameter can be accounted for by running a model multiple times. each time using a different value for the parameter (see Fig. 4.3). A large number of parameters can simulianeously be varied in this manner. using random draws for each run for each pa. raneter value, and the model run for hundreds, perhaps thousands, of times. As additional data become available, these uncertainiy distrihutions can be updated

At this print the modeller needs to provide probability dist ibutione for the values of the parameters under consideration. Hoffman and Kaplan (190;0) di cussing characterization of input parameters uncertainiw. nointed out that in of classical statistics (mean, variance) to $5 !$ mmmarize the variability of direct rit)

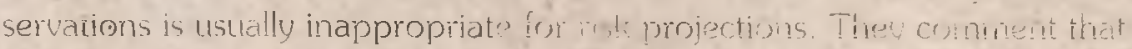

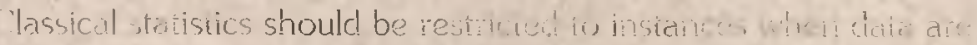

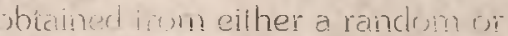




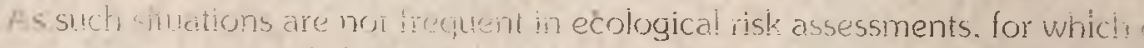
Ii tijulciluns are made beyond ine spatial exient and time periods in which dai have been collecied. combining e pert knowledge with sampling/experimenta

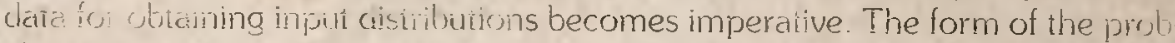
ability cistribution for any given barmeter value should reflect the knowlorlor of the irlue value. its varince. and oulincertainty in those values. For example a parametę aboui which nothing is known. except the probable range $|a \cdot b|$ could be assigned any value between $a$ and $b$ with equal probability (the uniform (a.b| conimuous disiribution). Alternatively. if there are maximum (a) anc minimum (b) values known. as well as a most likely value. a triangulai or ber probability distribution could be used (tig. 4.3). As many biological inpui variables are known to fit the normal or log-normal distribution. such distributions can be used if there is some knowledge of the mean and variance. Several at ternative probability distributions could also be used if there is sufficient uncleistanding of the parameters to justify their use. including skewed distribution (e.g. gànma. beia. Weibull) or discreie distributions (binomial, Poisson: known probabilities assigned to an enumerable set of possible values). For example. Miaia and Dourado-Neto (2004) used uniform, symmetrical triangular. and symmetrical truncated normal distributions for characterizing uncertainty of the resistance allele initial frequency, a key parameter in a simple, deterministic non-random mating mode! (Caprio. 1998).

Characterizing input distributions should be regarded as an iterative process: as parameters are identified where uncertainty is critical to model output. additional effort can be used to improve information on those parameters. For more details on the methods for obtaining distributions for uncertain model inputs, see Clemen and Winkler (1999), Hoffman and Kaplan (1999) and Kaplan (2000).

As an example of the Monte Carlo approach to uncertainty analysis, we consider below a very simple deterministic model of resistance evolution. This is a two-patch model, with one patch insecticidal (e.g. a Bt crop) and the other patch non-insecticidal (i.e. refuge), and resistance is conveyed by a single gene with two alleles. The insecticide is assumed to kill $100 \%$ of susceptible insects (SS genotype) and none of the resistant insects (RR genotype). Survival of heterozygotes (RS) is given by the functional dominance of the R allele, $h$. In ad: dition to $h$, there are two other parameters: the initial frequency of the $\mathrm{R}$ allele $\left(q_{0}\right)$ and the proportion of the landscape that is planted to the refuge $(x)$. The population consists of non-overlapping generations, there is no fitness cost associated with resistance, and there is random mating and random oviposition across the two patches (i.e. Hardy-Weinberg dynamics determine the frequen$c y$ of the three genotypes entering each generation). The model output is the $\mathrm{R}$-allele frequency after ten rounds of selection.

In the first instance, let us assume that nothing is known about the values of $h, q_{0}$, or $x$ : that is, we make no assumptions about the frequency of resistance, the functional dominance of resistance, or about how much the landscape will not be treated with the insecticide. Therefore, we assume that the values of 
each of the parameters are represented by uniform probability distributions between 0.0 and 1.0 . The model is run 1000 times, each with a new random independent draw for the values of the three parameters (i.e. Monte Carlo sampling procedure) using Crystal Ball ${ }^{\mathbb{B}} 2000$ (Decisioneering, Inc., Denver, Colorado). The probability distribution of the R-allele frequency after ten rounds of selection is given in Fig. 4.5a. The lowest final R-allele frequency is 0.0051 ; the highest is 1.0 . The mean is 0.85 , and the median is 0.95 . Seventy-five per cent of the runs result in a final R-allele frequency greater than $0.81 ; 25 \%$ of the runs result in a final $\mathrm{R}$-allele frequency greater than 0.99 . We conclude that a large proportion of the three-parameter space leads to rapid resistance evolution. However, we know that probability distribution across the parameter space is not uniform.

In a second iteration of the process, we make some assumptions about the parameter values based on what we know about use of $B t$ crops and resistance to the Cry proteins used in them to better reflect a real-world scenario. Bt cotton is planted on 60-90\% of cotton grown in the core of the US cotton belt (USDA NASS), varying by geography. Therefore the distribution of values for $x$, the proportion of the land as refuge (non-Bt cotton), can be better characterized by a uniform distribution with a minimum of $10 \%$ and a maximum of $40 \%$ (we do not have data giving the proportions of $B t$ and non-Bt on a finer scale than state level, preventing us from using a more refined probability distribution). The frequency of $R$ alleles to $B t$ cotton in tobacco budworm is believed to be extremely low, since years of monitoring for resistance and use of $B t$ cotton have not found any evidence of resistance. However, we do not believe it is zero. One literature estimate of R-allele frequency is $3 \times 10^{-1}$ (Gould et al., 1995b). Other modellers typically use a value of $10^{-3}$ or $10^{4}$ as an appropriate estimaie. We therefore modify our assumptions about the probability distribution of $\mathrm{R}$-allele frequency to be 10 , where $y$ is distributed normally with a mean of -3.5 and a standard deviation of 0.5 . The functional dominance of resistance to Bt cotton in tobacco budworm is also believed to be very low. High levels of resistance to Cry proteins tend to be recessive (Ferre and Van Rie. 2002), and Bt cotton expresses a high dose of Cry proteins such that heterozygotes are expected to show very lo survival. Functional dominance is unlikely to be zero, and the probability of a given value of $h$ is assumed to be distributed in log-normal manner with a mean value of 0.1 and siandard deviation of 0.05 .

The Mionte Carlo simulation (1000 ans) is conducted using these probability distributions for the three selected paraneters io obtain the probability distribution for $R$-allele frequency after ten rounds of selection (Fig. 5b). The distribution is somewhat bimodal, with a large peak at the lowest end of the range and a small peak at the highest end. The lowest final $R$ allels frequency is

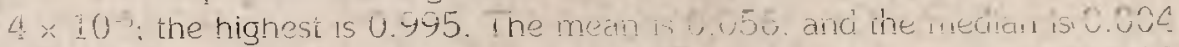
Three-quarters of the runs result in a fina! R-aliele frequency less than 0.02 The reñned probabilisic risk assessment vised on a very stin loteministic model indicates that the probability of usiance ir Bt cotton su ang in ten

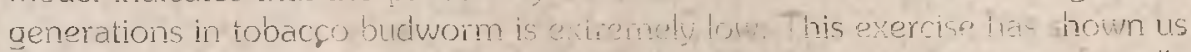

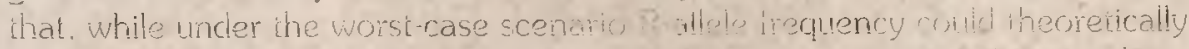

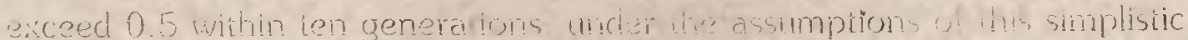


(a)

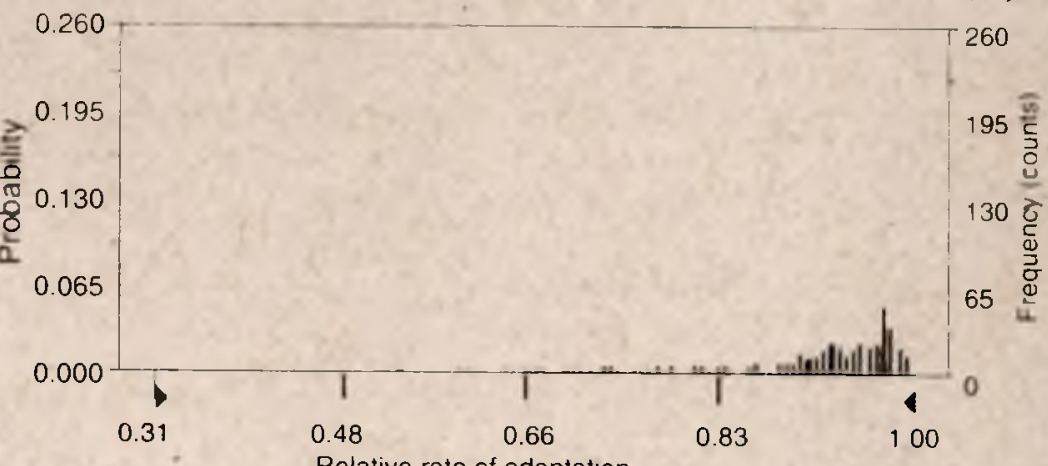

Relative rate of adaptation

1,000 Trials

(b)

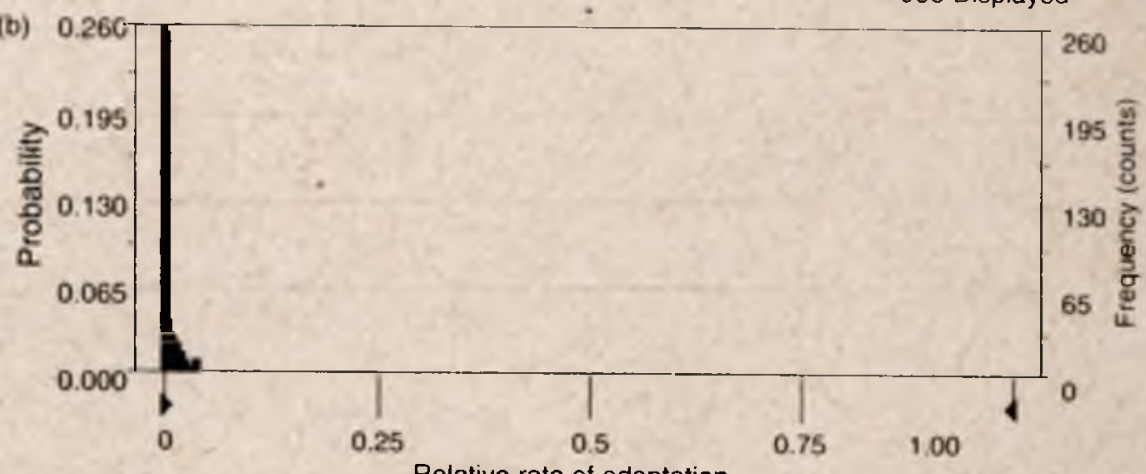

Relative rate of adaptation

2,000 Trials

60 Outliers

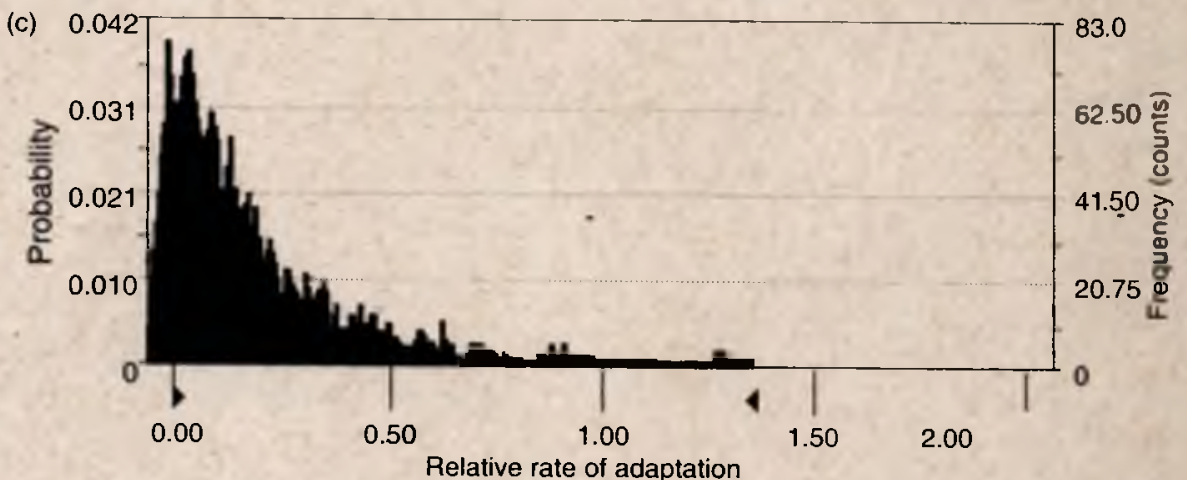

Fig. 4.5. Monte Carlo output distributions for resistance allele frequency after ten rounds of selection (i.e. 10-year gene frequency forecasts from 1000 trials) in a theoretical population based on Monte Carlo parameterization of a simple population genetics model with (a) no prior information and (b) prior, but uncertain, information on selection pressure, dominance of resistance, and initial allele frequencies.

Results from 958 trials are displayed in (a), 1000 in (b). 
model the probability of this occurring, based on understanding of resistance to $B t$ proteins and $B t$ cotton usage, is $<5 \%$.

Similar analyses can be applied to more sophisticated models of resistance evolution that incorporate more biologically realistic processes and more realistic agricultural landscapes. As more parameters are added to a model, it becomes important to consider how they may be correlated with one another. For example, a model may have two parameters governing insect dispersal: the proportion of individuals that disperse at a given time step and the distance that the dispersing insects move during the time step. These parameters may be negatively correlated; the rate of spread of a population across the landscape is likely to be a function of these two parameters. Thus in making random draws for one parameter, one may need to consider the random value drawn for the other, so that the rate of population spread remains realistic. In these situations, we need to use methods for sampling input distributions that account for correlation patterns among parameters (e.g. Latin hypercube sampling) (Iman and Conover, 1982).

These risk assessment techniques also offer advantages in the area of sensitivity analysis. If the randomly assigned parameter values are saved along with the simulation results, a sensitivity analysis that includes interactions and correlations among parameters can be performed. Assuming the data can be linearized, step-wise regression or Bayesian model averaging can be used to identify the parameters or parameter interactions the model is most sensitive to (Caprio et al. . 2006; McCaffery et al. . 2006). These interactions are generally left out in conventional scenario-based modelling and sensitivity analysis. For example, in a data set examining the introduction of Vip3A cotton (Caprio and MicCaffery, unpublished data), the data were first analysed without interactions between parameters, which showed significant effects for initial gene frequency and dominance of the resistance allele. When all two-way interactions were included into the model, a highly significant interaction term between these two

- parameters was found. In retrospect, this interaction is reasonable. As we know from basic population genetics texts, the heritability of recessive traits increases diamatically as gene frequency increases, and thus the impaci of doininance decreases as initial gene frequency approaches 1.0

Using a version of a deterministic non-random mating model (Caprio ei al. 1998). Miaia (2003) found complex interactions in a sensitivity analysis. She analysed the sensitivity of two output variables. R-allele frequency acioss targetpest generations (RFreq) and number of generations until resistance (NGei). to perturbations in the input parameiers initial R-allele frequency and functinna! dominance of resistance. for different scenarios (combinations of eivge size and refuge pest management). The results showed that RFreq sensitivity to both input variables changed considerably among scenarios as well as across generations, ranging from high sensitivity (e ponential patterns) in the initial jenn. ations to null sensitivity after several rounds of selection.

The model of Western con rontworm adaptation to Bt maizo in strorer (2003) was subjected io just such an analysis (Storer. unpublished daia). or ihis vinte Carlo sensitivily analysin. 27 parameters were simultaneously varied acording io preclefined probability distributions based on assurnptinne of the. 
underlying distribution of uncertainty or variability for each parameter, and the model was run 2000 times. Two parameter correlations were included. First. sur vival of susceptible larvae on $B t$ maize was $80 \%$ positively correlated with the functional dominance (and therefore with the relative survival of heterozygous larvae). Secondly. the fecundity of adult rootworms was $80 \%$ negatively correlated with density-independent mortality in winter, so that the population size in the absence of $\mathrm{Bt}$ maize remained biologically reasonable. The output from the sensitivity analysis provided a distribution for expected rates of adaptation for rootworms when Bt maize was planted on $90 \%$ of the acreage (10\% refuge). For this model. a relative rate of adaptation $(R R A)$ was defined as the average annual increase in $\mathrm{R}$-allele frequency on a log scale, expressed as a fraction of the baseline rate of adaptation:

$$
\mathrm{RRA}=\left(\frac{1}{Y} \ln \frac{q_{Y}}{q_{0}}\right) / 0.327
$$

where $q_{y}$ is the $\mathrm{R}$-allele frequency after $Y$ years and 0.327 is the adaptation rate (year ${ }^{1}$ ) for the baseline run (Storer, 2003). This rate is calculated when the $\mathrm{R}$ allele frequency first exceeds 0.075 , when the rootworm egg population in the autumn falls below 20,000 per field, or after 10 years, whichever is soonest.

The mean RRA for the Monte Carlo analysis was 0.35 (standard deviation $=0.42$ ), while the median was 0.23 (Fig. 4.5c). These averages represent 3.5 to 5.4 times slower adaptation than that predicted using the default parameter settings. The extreme values were -0.86 and 5.07 (negative values of RRA resulted from a decline in region wide $r$-allele frequency due to local $r$-allele extinction when populations were small). In the sensitivity analysis, $96.5 \%$ of the RRA values for the same value were less than 1.24 . This finding suggests that the RRA value obtained using the default parameters is greater than the $95 \%$ confidence limit for the estimate of the true RRA.

The parameters with the largest effects on RRA were the functional dominance of the $\mathrm{R}$ allele (explaining $58.5 \%$ of the variation) and the dose (explaining $33.5 \%$ of the variation). Lower functional dominance and higher doses caused lower RRA. As input parameters, these two parameters had $80 \%$ correlation. The next most important parameter was another genętic factor: the fitness cost of resistance (3.1\% of the variation). Winter survival $(2.9 \%)$ and $f e-$ cundity (1.1\%), the pair of correlated parameters, were the next most important. The other parameters combined explained less than $1 \%$. These findings indicate that, until we have isolated resistance alleles in the field, it will be difficult to predict with greater accuracy the rate that the alleles will spread. The probability that adaptation will be slower than predicted by using default values was greater that $95 \%$, suggesting that the set of default parameter values chosen in this case was highly conservative.

Caprio et al. (2006) incorporated both stochastic influences and parameter uncertainty in a model of resistance to methyl parathion in Western corn rootworm. They compared the variance in model results (Monte Carlo output distribution for 'time until resistance', TR) with parameters fixed at their default values and estimated the impact of stochastic influences. Additional runs of the 
model integrated both parameter uncertainty in 18 parameters and stochastic influences to estimate their joint influences. In this case, the parameter uncertainties were defined as normal distributions with a standard deviation that was a specified percentage of the default mean value. Uncertainty was not included in Fig. 4.4a. The variability among model output values was entirely due to stochastic demographic processes. For the other cases, the standard deviation increased from 5 to 10 to $20 \%$ (Fig. $4.4 \mathrm{~b}$, c, and d) demonstrating the impact of increased parameter uncertainty on the TR Monte Carlo output distribution. Ultimately, this combination of both stochastic and parametric uncertainty provides an improved description of the model output uncertainty, thus improving our ability to predict the time it will take for resistance to evolve to a particular insecticidal protein in a particular use pattern.

Resistance risk assessment requires that the modeller not only provides point estimates for input parameters, but also a complete characterization of their respective uncertainties via probability distribution functions (PDFs). These PDFs can come from a number of sources, including expert opinion and empirical data (Hoffman and Kaplan, 1999; Kaplan, 2000). In the worst scenario, a flat uniform distribution can be chosen to describe uncertainty of parameters for which there is no prior information. In cases where there is a minimum of data to support a default parameter value, many modellers will be reluctant to speculate on an input probability distribution. Often, however, limits to likely values can be estimated which, together with a most likely value (usually the default value), provide sufficient information for a triangular distribution. The beta distribution can also be used with these three parameters, avoiding the sharp changes in the second moment present in the triangular distribution! The beta distribution also places less emphasis on the tails of the distribution than the triangular distribution. Vose (2000) describes a PERT distribution based on the beta distribution, as well as a modified PERT that reduces the weight placed on the most likely value, gradually transforming into a uniform distribution. The beta distribution requires four parameters: a minimum and maximum value as well as two shape parameters. The modified PERT distribution uses the most likely value and a bias value (defaulting to four in the standard PERT distribution) to determine the two shape parameters. This modified PERT distribution can be used to reflect the modeller's uncertainty in his/her estimate of the most likely value. These distributions require a minimum number of parameters (maximum. minimum, and most likely values) and provide a simple mechanism to incorporate uncertainty into models. Subsequently. sensitivity analysis can indicate which parameters have the most impact on the model results. and further refinements can be made in the uncertainty characterizations for those parameters

It should be realized that risk estimates can be highly sensitive to the iype

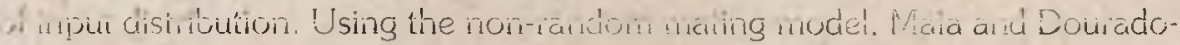
Neto (2004) evaluated the influence of three types of distributions for the R-allele initial frequency: uniform (UN), symmetical triangular (ST), and symmetrical muncated mormal $(T N V)$, with the same range nn risk estimates (probability of RFreq excerding a critical value) across grinerations (or three resistance manhomen! scenarios. For all scenarios thei foulind that risk stimates comespon 


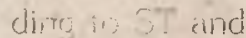

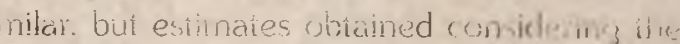

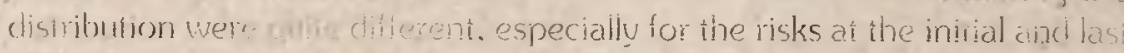
uris of selection

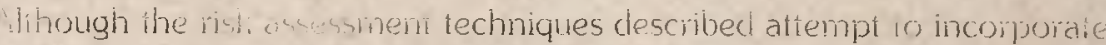
ail c citable knowledge in in for malized framework the uncertainiy distributions will ofien contain elemenis of subjectivity. While the results are often urst. sen sitive to small variations in the maximum or minmum values in a iriangulai dis. tribution (these are cireas it probability). humans tend io underesimaie ist: (Vose. 2000: Bedford and Gooke. 2001: Evans and Olsen, 2002). and hence sei these exireme values tou conservatively. Of particular interest is the process of inchoring (Vose. $20(1)$ ). in which the modeller anchors his/her estimate to a most likely value and seis critieme values based on this estimate. This usually underestimates uncertamy. and extreme values should be independentu estimated. In general. the process of collecting and combining expert opinion re quires great attention from the modeller.

In some cases. because of the large number of parameler combinations. it may be difficult to summarize al! results using a single criterion, and all combinations may not be reasonable. For example. in Fig. 4.4a. b, c and d (Caprio et al. 2006), one can see a bimodal distribution developing as the uncertainty and observed frequencies of field failures within 3 years increase. In this case. as the variance in the input distributions increased. an increasing number of simulations included parameter combinations that effectively did not control the simulated pest. and the model results reflected only the time required for the initial population to build up to damaging levels. In a subsequent analysis. these values were excluded. because it was presumed that the pesticide never would have been deployed under those settings. Another example is the determination of proper stop criteria that are suitable for all realizations of a model. Most modellers use some sort of frequency-based criterion, for example when the resistance allele frequency in relevant fields exceeds $50 \%$. However, when performing a risk assessment, some of the simulations may include parameters that lead to extremely low rates of selection, and the simulations are generally stopped after a reasonably long period of time (20-50 years). This leads to right censored data with little resolution in how changes in parameters affect resistance evolution beyond the maximum simulated time. Under some situations, this could lead to an underestimate of the sensitivity of the model to certain parameters. Approaches using performance criteria (the ability of the toxin to maintain populations below some level) (Caprio et al., 2006) are also subject to similar problems. An alternative approach is to estimate the exponential rate at which the resistance allele frequency changes per year and to use this as a qualitative measure of risk (e.g. Storer, 2003). The assumption here is that the rate of change in allele frequency is constant as long as resistance alleles are rare (Fig. 4.6). This rate is determined almost entirely by the fitness of resistant heterozygotes relative to that of susceptible homozygotes, and the rate only begins to change when resistant homozygotes begin to be relatively common. As an aside, note that one can change the fitness of the homozygote considerably, changing dominance from recessive to dominant, while having little impact on the rate of resistance evolution. Dominance is relevant only to the degree it describes the 


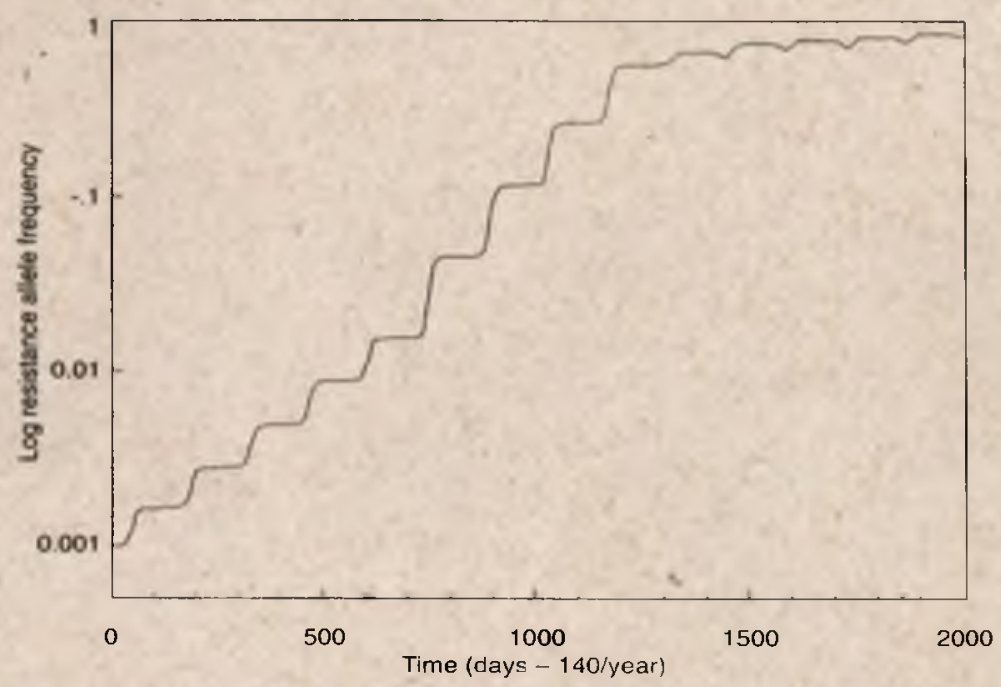

Fig. 4.6. The constant rate of increase in log resistance allele frequency in a simulation of Western corn rootworm resistance to a low-dose transgenic event.

relative fitness of heterozygotes or susceptible homozygotes. The estimated rates can have problems when initial gene frequencies are varied due to uncertainty. In a model of the introduction of Vip3a cotton. Caprio and McCaffery (unpublished data) varied the initial gene frequency from $10^{1}$ to $10^{-4}$, based on published estimates for other species. In realizations of the model where the initial frequency was close to $10^{-1}$. the resistance allele frequency was high enough that it no longer fell within that region where the exponential rate of increase was constant. In these cases the rate could not be reliably estimated, leading to left censored data. Other realizations of the model used parameter values that would lead to long resistance times (indeed, with fitness costs, negative rates can easily be achieved). so frequency-based criteria would also lead to loss of information due to right censored data. It may not be unusual that the extensive variety of results in a risk assessment will require that some information be lost. but the results should indicate the important parameters within the time frame examined.

Resistance simulations. especially prospective ones run before widespread deployment of a new technology, tend to be ruin assuming maximum adoption of the technology. While it is possible to predict technology adoption curves, this is rarely done, meaning that the results are highly conservative. One outcome of this approach may be to magnify the effects of parameter uncertainty on the predictions of resistance eunituinn. 


\section{Conclusions: Towards the Appropriate Interpretation and Use of Resistance Models}

As resistance risk assessment and management has developed since the 1950s, especially spurred on by the release of transgenic insecticidal crops, we have seen enormous reliance on models. Empirical experimentation with insect resistance is inherently tricky, since a successful field experiment to examine the effectiveness of a given strategy would inevitably create the problem we are trying to avoid. Experiments in greenhouses (Tang et al, 2001: Zhao et al., 2003) can be regarded as models of the field in that the environmental influences are controlled, as are many of the biological processes along with the operational manipulations. Data from such experiments have been extremely useful in validating the findings of the simple generalized computer models, which can be regarded as experiments in cyberspace. From these physical and virtual experiments, we have been able to assess the relative merits of different resistance management strategies, such as high-dose/refuge and gene pyramiding. However, it remains problematic to extend these findings to devise appropriate strategies to use in commercial field conditions.

More complex models are more system-specific and have greater predictive utility to understand the resistance risks in any particular use pattern. However, as we have seen, it is vital to understand the predictive limitations of these models, particularly by understanding the uncertainty associated with the model predictions. 'Garbage in, garbage out' is a truism of these models, and modellers are faced with a huge challenge to parameterize them correctly. Indeed, there is generally no single correct parameter set to use when modelling across space and time.

Crude attempts to capture the uncertainty by running best-case and worstcase scenarios are of limited utility if there is no attempt to relate those scenarios to the real world. In this chapter, we have described the application of established risk assessment tools to resistance management models that provide more useful descriptions of our uncertainties in predictions of resistance evolution. While simple models have been used to develop regulatory policy around resistance management (ILSI/HESI, 1999; US EPA, 2001; Fitt et al., 2006), we are seeing with more sophisticated approaches that not all the important processes are necessarily taken into account, and that the resistance risk is not uniform for all products and all use patterns. That resistance has not evolved to $B t$ crops after more than 10 years of use is evidence that one or more of the assumptions made in the early models were highly conservative or that those models inadequately described the resistance risks (Tabashnik et al., 2003).

System-specific models with an appropriate uncertainty analysis in a risk assessment context show whether or not resistance management strategies are needed, and, if so, what strategies should be implemented. Clearly, the need for flexibility inherent to this approach does not lend itself to a 'one size fits all' regulatory-driven strategy, as much as it does to a locally developed and implemented strategy within an overall regulatory policy. With probabilistic risk assessment tools, we can start to determine under what circumstances there 
may be an unreasonable resistance risk, and consequently under what circumstances resistance management is warranted. Of course, for implementation of the risk assessment process to be useful, there must be some agreement of what constitutes an unacceptable risk. By leaving this undefined, the European and Mediterranean Plant Protection Organization has left the interpretation of unacceptability to the subjective opinion of regulatory decision makers (Leonard, 2000). For resistance to $B t$ crops, this could be failure of the product within a certain time frame with a certain probability. With spatial models, this could be further refined by stating over what proportion of the area where the Bt crop is used resistance would be unacceptable. A regulatory policy developed around defining these unacceptable risks would go a long way towards establishing rational resistance management plans.

\section{References}

Alstad, D.N. and Andow. D.A. (1995) Managing the evolution of insect ressistance to transgenic plants. Science 268, 1894-1896.

Barber, G.W. (1936) The Cannibalistic Habits of the Corn Ear Worm. Technical Bulletin No. 499. US Department of Agriculture, Washington, DC

Bedford, T and Cooke, R. (2001) Probabilistic Risk Analysis: Foundations and Methods. Cambridge University Press, Cambridge, UK.

Caprio. M.A. (1994) Bacillus thuringiensis gene deployment and resistance management in single and multi-tactic environments. Biocontrol Science and Technology 4, 487-497.

Caprio, M.A. (1998) The evolution of resistance: a simple deterministic model. http://www.msstate.edu/Entomology/PGjava/ILSImodel.html (accessed December 2007).

Caprio, M.A. (2001) Source-sink dynamics between transgenic and non-transgenic habitats and their role in the evolution of resistance. Journal of Economic Entomology 94, 698-705.

Caprio. M.A. and Tabashnik. B.E. (1992) Gene flow accelerates local adaptation among finite populations: simulating the evolution of insecticide resistance. Journal of Economic Entomology 85, 611-620

Caprio. M.A. Faver. M.K. and Hankins, G. (2004) Evaluating the impacts of refuge width on source-sink dynamics between transgenic and non-transgenic habitats. Journal of insect Science 4. 3.

Caprio. Mi.A. Nowatzki. I. Siegfried. B., Venke, L.J. Wright, R.J. and Chandler, L.D. (2006) Assessing risk of resistance to aerial applications of methyl-parathion in western corn rnotworm (Coleoptera: Chrysomelidae). Joumal of Economic Entomology 99. 483-493

Camière. Y.P. Dutilleul. P. Ellers-Kirk. C. Pedersen, B Haller. S. Antilla L.. Dennehy T.j. and Tabashnik. B.E. (2002) Sources, sinks, and the zone of influence of refuges for managing insect resistance to Bt crops. Ecological Applications 14. 1615-1623

Chapman. P.E. (1982) The Insects: Structure and Function. Harvard University Press. Cambridge. Miasiachusetts.

Clemen. R.T. and Winkler. R.L. (1999) Combining probability distributions fiom experts in risk makisis Risk Andusis 10 $187-203$

Comins. 11 11977a) The development of insecticide rosistance in the presence of migration Journal of Theoretion Binlogy 64. 177-107

Comms. If.N. (1977b) The management of pesticide resistance. Joumal of Theoretical Biolouy $65.390-120$

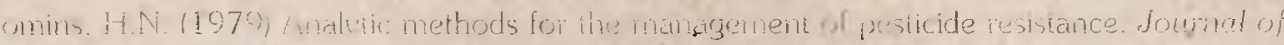

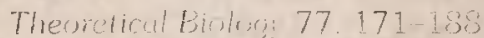




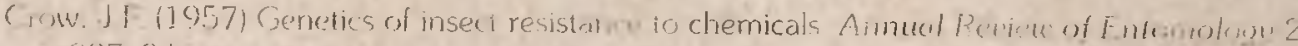
$227-24 \%$

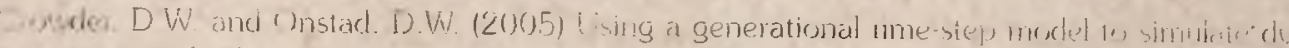

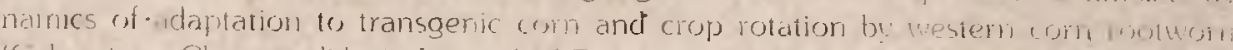

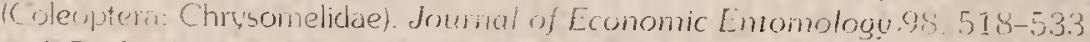

Curtis. CF Cook. L.M. and Wood. R.j. (1973) Selection for and agains insecticider resistance and pussible methods of intmbining the evolution of resistance in mosquitoes. Ficolouscal Entomologe 3. 27.3-287

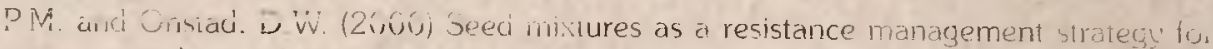
European corn borers (Lepidoptera: Crambiclae) infesting transgenic corn expressing Civ $1 \mathrm{AU}$ protein. Journal of Economic Entomology 93. 937-948.

Evans. J.R. and Olsen. D.L. (2002) hintroduction to Simulation and Risk Andusis 2ind edi Prentice-Hall. Upper Sactdle River. New Jersey.

Firé. F and Van Rie. J. (2002) Biochemistry and genetics of inseci resistance to Bacillus thuringiensis. Annual Review of Eniomology 47.501-533

Fitt. G.P. Omoto. C.. Maia. A.H.N.. Waquil. J.M.. Caprio. M.. Okech. M.A.. Cia. E.. Huai N.H. and Andow. D. (2006) Resistance risks of Bt cotton and their management in Brazil environmenta! risk assessment of genetically modifiect organisms. In Hilbeck. A. Andow. D. and Fontes. E.M.G. (eds) Methodologies for Assessing Bt Cotton in Brazil. Vol 2 CAE International. Wallingford, UK. pp. 300-345.

Georghiou. G.F. and Taylor, C.E. (1977a) Genetic and biological influences in the evolution of in secticide resistance. Journal of Economic Entomology 70. 319-323.

Georghiou. G.P and Taylor, C.E. (1977b) Operational influences in the evolution of insecticide resistance. Journal of Economic Entomology 70, 653-658.

Gould. F. (1986) Simulation models for predicting durability of insect-resistant germplasm: hessian fly (Diptera: Cecidomyiidae)-resistant winter wheat. Environmental Entomology 15. 11 23

Gould. F. (1994) Potential problems with high-dose strategies for pesticidal engineered crops Biocontrol Science and Technology 4, 451-462

Gould. F. (1998) Sustainability of transgenic insecticidal cultivars: integrating pest genetics and ecology. Annual Review of Entomology 43. 701-726

Gould, F., Kennedy, G.G. and Johnson, M.T. (1991) Effects of natural enemies on the rate of her bivore adaptation to resistant host plants. Entomologia Experimentalis et Applicata 58, 114.

Gould. F. Anderson, A.. Reynolds, A.. Bumgarner. L. and Moar. W. (1995a) Selection and genetic analysis of a Heliothis virescens (Lepidoptera: Noctuidae) strain with high levels of resistance to Bacillus thuringiensis toxins. Journal of Economic Entomology 88, 1545-1559.

Gould, F., Anderson, A., Jones, A., Sumerford, D., Heckel, D.E., Lopez, J., Micinski, S., Leonard, R. and Laster, M. (1995b) Initial frequency of alleles for resistance to Bacillus thuringiensis toxins in field populations of Heliothis virescens. Proceedings National Academy of Science USA 94, 3519-3523.

Guse, C.A., Onstad, W., Buschman, G.L.L., Porter, G.P., Higgins, R.A., Sloderbeck, P.E.. Cronholm, G.B. and Peairs, F.B. (2002) Modeling the development of resistance by stalkboring Lepidoptera (Crambidae) in areas with irrigated transgenic corn. Environmental Entomology 31, 676-685.

Gustafson, D.I., Head, G.P. and Caprio, M.A. (2006) Modeling the impact of alternative hosts on Helicoverpa zea adaptation to bollgard cotton. Journal of Economic Entomology 99. 2116-2124

Hoffman, F.O. and Kaplan, S. (1999) Beyond the domain of direct observation: how to specify a probability distribution that represents the state of knowledge about uncertainty inputs. Risk Analysis 19, 131-134. 
ILSI, HESI (1999) An Evaluation of Insect Resistance Management in Bt Field Corn: A ScienceBased Framework for Risk Assessment and Risk Management. Report of an Expert Panel. International Life Sciences Institute/Health and Environmental Sciences Institute. Washington, DC.

Iman, R.L. and Conover, W.J. (1982) A distribution free approach to inducing rank correlations among input variables. Communications in Statistics: Simulation and Computation 11 , 311-334.

Isukapalli, S.S. and Georgopoulus, P.G. (2001) Computational Methods for Sensitivity and Uncertainty Analysis for Environmental and Biological Models. http://www.ccl.rutgers.edu/reports/EPA/edmas_v3_epa.pdf (accessed November 2007).

Jurat-Fuentes, J.L., Gould, F.L. and Adang, M.J. (2000) High levels of resistance and cross-resistance to Bacillus thuringiensis Cry1 toxins in Heliothis virescens are due to reduced toxin binding and pore formation. Resistant Pest Management 11, 23-24.

Kaplan, S. (2000) Combining probability distributions from experts in risk analysis. Risk Analysis 20, 155-156.

Kennedy, G.G. and Storer, N.P. (2000) Life systems of polyphagous arthropod pests in temporally unstable cropping systems. Annual Review of Entomology 45, 467-493.

Lenormand, T. and Raymond, M. (1998) Resistance management: the stable zone strategy. Proceedings of the Royal Society of London. Series B 265, 1985-1990.

Leonard, P.K. (2000) Resistance risk evaluation, 'a European regulatory perspective'. Crop Protection 19, 905-909.

Mallet. J. and Porter. P. (1992) Preventing insect adaptation to insect-resistant crops: are seed mixtures or refugia the best strategy? Proceedings: Biological Sciences 250. 165-169

Miaia, A.H.N. (2003) Modelagem da evolução da resistência de pragas a toxinas Bt expressas em culturas transgênicas: quantificação de risco utilizando análise de inceltezas. PhD thesis. Universidade de São Paulo. Brazil: http://www.teses.usp.br/teses/disponiveis/ $11 / 11136 /$ tde-19012004-100211

Maia. A.H.N. and Dourado-Neto, D. (2004) Probabilistic tools for assessment of pest resistance risk associated to insecticidal transgenic crops. Scientia Agricola 61 48 485.

McCaffery. A. Caprio. M.. Jackson, R., Marcus. M.. Martin, T., Dickerson, D. Negrotto. D. O Reilly. D.. Chen. E and Lee, M. (2006) Effective IRM with the novel insecicicidal protein Vip3A. In: Proceedings of the 2006 Beltwide Cotton Production Research Conference. National Cotton Council. San Antonio. Texas.

McGaughey. W.H. and Whalon. Mi.E. (1992) Managing insect resistance to Bucillus thuringien. sis toxins. Science 258, 1451-1455

" tetcalf. R.L. (1973) A century of DDT. Journal of Agriculture and Food Ch:mistru 21. 511 519

Onstad. D.W. and Gruld. F. (1998) Modeling the dynamics of adaptation (1) Mansgenic maize by Eropean com bre! (Lepidoptera: Pyralidae). joumal of Economic Enimolugu 31.585 593

Onstad. D.W. Guse. CA. Spencer, J.L., Levine. E. and Grav. MI.E. (20!) I) Virieling the dy namics of adajtation to transgenic com by western corn mut mim lColersptera Chrysomelidaes Journal of Economic Entomology 94. 529-540.

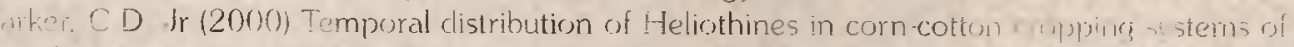

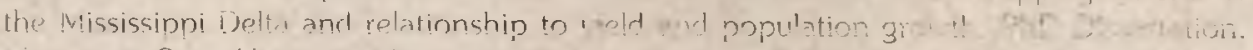
Mississippi State University. Mississippi State. Mi sissippi.

S.L. (2004) Simulation a cepperiment: a philosciphical reassessment

ing Trends in Ecolowe of Exolution $19.530,534$

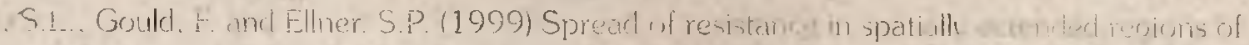

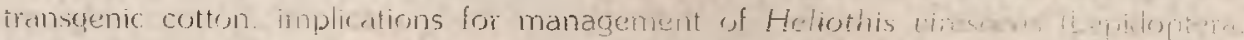

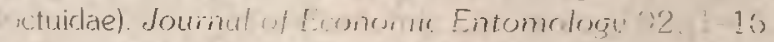




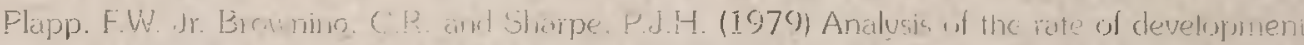

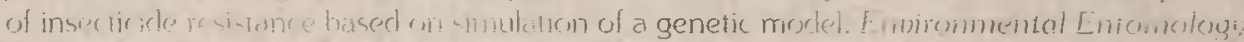
(1) 40,500

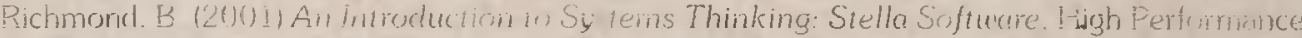
Sustems. Itanover New Hanushire.

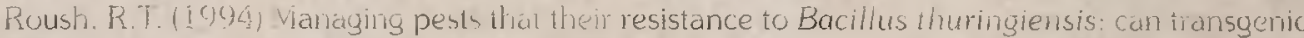
crops the better than spraus? Birsuntrol Science and Technology 4.501-516.

Siqueira. H.A. vinellenbeck. D. Spencer. 'T. and Siegfried. B.D. 12004 ) Cross-resistance of

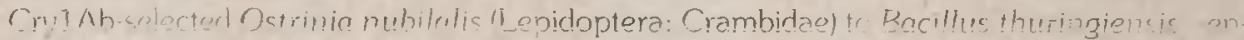
doto.ins. Joumal of Economic Entomology 97. 1049-1057

Sisterson. vi.S.. Antilla. L... Carriere. Y.. Ellers-Kirk, C. and Tabashnik. B.E. (2004) Effect of insect population size on evolution of resistance to transgenic crops. Joumal of Economic Entomologu $37.1413-1424$

Sisteison. Vi.S. Carriere. Y. Dennehu T.J. and Tabashnik. B.E. (2005) Evolution of resistance to transgenic crops: interactions between insect movement and field distribution. Jormul of Economic Entomology 98 . 1751-1762

Stadelbacher. E.A. (1979) Geranium dissectum. an unreported host of the tobacco budursm and bollworm and its role in their seasonal and long term population dynamics in the delia of Mississippi. Environmental Eniamology 8, 1153-1156

Siorer. N.F. (2003) A spatially explicit model simulating western corn rootworm (Coleoprera: Chrysomelidae) adaptation to inseci-resistant maize. Journal of Economic Entomologi 96. $1530-1547$

Storer. N.P. Peck. S.L.. Gould. F. Van Duyn. J.W. and Kennedy, G.G. (2003a) Spatial processes in the evolution of resistance in Helicoverpa zea (Lepidoptera: Noctuidae) to Bt transgenic corn and cotton in a mixed agroecosystem: a biology-rich slochastic simulation model. Journal of Economic Entomology 96. 156-172.

Storer. N.P.. Peck. S.L.. Gould. F.. Van Duyn. J.W. and Kennedy, G.G. (2003b) Sensitivitu analy. sis of a spatially-explicit stochastic simulation model of the evolution of resistance in Helicoverpa zea (Lepidoptera: Noctuidae) to Bt transgenic corn and cotton. Journal of Economic Entomology 96. 173-187

Tabashnik. B.E. (1986) Computer simulation as a tool for pesticide resistance management. In. Pesticide Resistance: Strategies and Tactics for Management. National Academy Press. Washington, DC, pp. 194-206.

Tabashnik. B E. (1994a) Delaying insect adaptation to transgenic plants seed mixtures and refugia reconsidered. Proceedings of the Royal Society of London. Series B 255, 7-12.

Tabashnik. B.E. (1994b) Evolution of resistance to Bacillus thuringiensis. Annual Review of Entomology 39, 47-79.

Tabashnik. B.E. and Croft, B.A. (1982) Managing pesticide resistance in crop-arthropod complexes: interactions between biological and operational factors. Environmental Eatomology $11,1137-1144$.

Tabashnik. B.E. and Croft, B.A. (1985) Evolution of pesticide resistance in apple pests and their natural enemies. BioControl 30, 37-49.

Tabashnik. B.E., Carrière, Y., Dennehy, T.J., Morin, S., Sisterson, M.S., Roush, R.T., Shelton, A.M. and Zhao, J.Z. (2003) Insect resistance to transgenic Bt crops: lessons from the laboratory and field. Journal of Economic Entomology 96, 1031-1038.

Tang, J.D., Collins, H.L., Metz, T.D., Earle, E.D., Zhao, J.Z., Roush, R.T. and Shelton, A.M. (2001) Greenhouse tests on resistance management of $B t$ transgenic plants using refuge strategies. Journal of Economic Entomology 94, 240-247.

Taylor, C.E. (1983) Evolution of resistance to insecticides: the role of mathematical models and computer simulation. In: Georghiou, G.P. and Saito, T. (eds) Pest Resistance to Pesticides. Plenum Press, New York, pp. 163-173. 
Taylor, C.E. (1986) Genetics and the evolution of resistance to insecticides. Biological Journal of the Linnean Society 27, 103-112.

Taylor, C.E. and Georghiou, G.P. (1979) Suppression of insecticide resistance by alteration of gene dominance and migration. Journal of Economic Entomology 72, 105-109.

Taylor, C.E. and Georghiou, G.P. (1982) Influence of pesticide persistence in evolution of pesticide resistance. Environmental Entömology 11, 746-750.

Taylor, C.E. and Georghiou, G.P. (1983) Evolution of resistance insecticides: a case study on the influence of migration and insecticide decay rate. Journal of Economic Entomology 76 , $704-707$.

US Department of Agriculture, National Agriculture Statistics Service USDA NASS. Quick Stats: Agricultural Statistics Database. http://www.nass.usda.gov/QuickStats

US EPA (2001) Biopesticides Registration Action Document - Bacillus thuringiensis PlantIncorporated Protectants. D. Insect resistance management. http://www.epa.gov/pesticides/biopesticides/pips/bt_brad.htm (accessed November 2007).

Vose, D. (2000) Risk Analysis: A Quantitative Guide. Wiley \& Sons, New York. -

Wearing, C.H. and Hokkanen, H.M T. (1995) Pest resistance to Bacillus thuringiensis: ecological crop assessment for $\mathrm{Bt}$ gene incorporation and strategies of management. In: Hokkanen, H.M.T. and Lynch, J.M. (eds) Biological Control: Benefits and Risks. Cambridge University Press, Cambridge, UK. pp. 236-252.

Zhao, J.Z., Cao, J., Li, Y., Collins, H.L., Roush, R.T., Earle, E.D. and Shelton, A.M. (2003) Transgenic plants expressing two Bacillus thuringiensis toxins delay insect resistance evolution. Nature Biotechnology 21, 1493-1497. 